\title{
Theoretical and experimental studies of highly selective planar two-dimensional Bragg structures based on dielectric waveguides in the terahertz frequency range
}

\author{
Naum Ginzburg, Nikolay Peskov, Vladislav Zaslavsky, Ekaterina Kocharovskaya*, \\ Andrey Malkin, Alexander Sergeev, Michael Proyavin, and Dmitry Sobolev
}

Institute of Applied Physics RAS, 46 Ul'yanov St, 603950, Nizhny Novgorod, Russia

\begin{abstract}
Based on theoretical approach and three-dimensional modeling using the CST Microwave Studio code, planar dielectric two-dimensional Bragg structures in terahertz frequency range were developed and manufactured. Proof-of-principle electrodynamic experiments on the "cold" testing of these structures were carried out. It is shown that the experimental results are in good agreement with the theoretical predicts, including the existence of the highest Q mode inside the Bragg reflection band in the absence of periodicity defects.
\end{abstract}

\section{Introduction}

The use of two-dimensional distributed feedback (2D DF) has been proposed in Refs. 1 and 2 as a method of producing spatially coherent radiation from either sheet or annular highcurrent relativistic electron beams with the transverse size greatly exceeding the wavelength. In this case, such feedback can be realized in 2D Bragg metallic cavities of planar and coaxial geometry having a double-periodic corrugation of the side walls. On this corrugation, mutual scattering of the electromagnetic energy fluxes propagating in the forward, backward and transverse directions (relative to the direction of the electron beam propagation) takes place. These transversely propagating waves act to synchronize radiation from different parts of the spatially extended active medium. To date, the operability of the new feedback mechanism has been experimentally demonstrated in the FEMs, which were elaborated in the millimeter wavelength range (from Ka- up to W-bands) under a record transverse size of the interaction space, reaching up to 50 wavelengths, and the output power level of $\sim 50-100 \mathrm{MW}[3,4]$.

In this paper, we consider the possibility of expanding the class of oscillators using 2D $\mathrm{DF}$, as applied to quantum oscillators, including heterolasers. One of the attractive ways to solve mode selection problem in heterolasers is the use of novel 2D feedback mechanism, which in this case can be realized by 2D Bragg structures of planar geometry with doubleperiodical modulation of the effective refractive index of the dielectric waveguide. It should be noted that in lasers based on semiconductor active media, including semiconductor

* Corresponding author: katya@appl.sci-nnov.ru 
heterostructures [5], planar geometry is a consequence of the epitaxial-lithographic manufacturing technology. According to the theoretical analysis carried out in [6], the use of these structures makes it possible to synchronize the radiation from the active medium characterized by large Fresnel parameters in both transverse directions:

$$
F_{x}=l_{x}^{2} / \lambda l_{z}>>1, F_{z}=l_{z}^{2} / \lambda l_{x}>>1 .
$$

The paper presents result of theoretical analysis and computer simulations of the electrodynamic properties of dielectric 2D Bragg structures of planar geometry. "Cold" tests of prototypes of the structures were carried out in terahertz frequency range and demonstrated good coincidence with the design parameters.

(a)

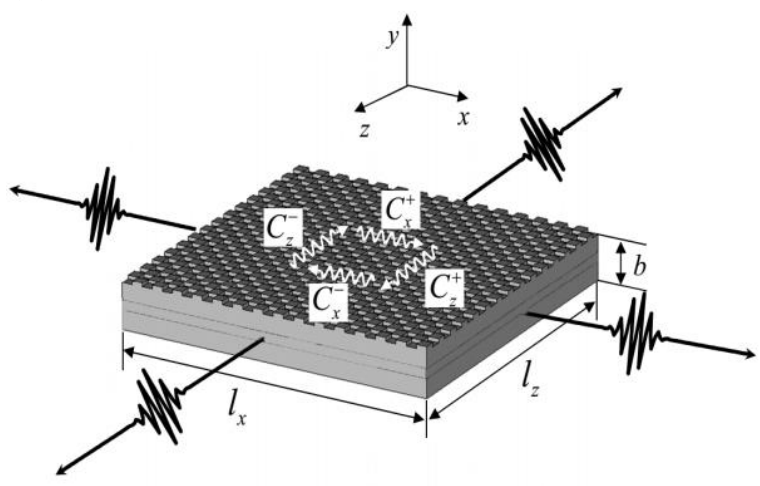

(b)

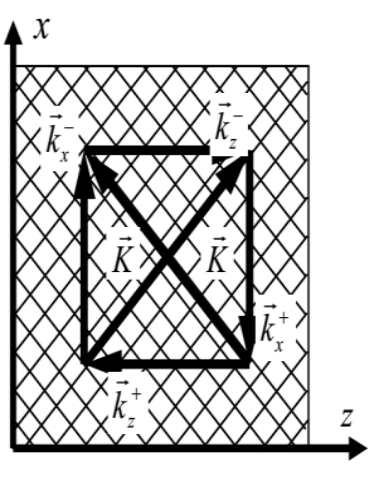

Fig. 1. (a) Scheme of 2D Bragg resonator based on a planar dielectric waveguide with double-periodical modulation of the effective refractive index. Wavy arrows indicate the directions of partial wave beams bound to the 2D Bragg structure. (b) Diagram illustrating the scattering of partial waves ( $\vec{k}_{x, z}^{ \pm}$- wave vectors of the partial waves, $\vec{K}^{ \pm}=\vec{h} \vec{x}_{0} \pm \vec{h} \vec{z}_{0}$ - translational vectors of the two-dimensional grating).

\section{Eigenmodes of the planar 2D Bragg resonator}

Let us consider the following model of a $2 \mathrm{D}$ Bragg resonator, assuming that twodimensional-periodic corrugation is applied to a rectangular area of one of the surfaces of a dielectric plate with the result that the plate thickness is modulated by the law (Fig. 1)

$$
b(x, z)=b_{0}+b_{1}\left(\cos \left(\bar{h}_{x} x+\bar{h}_{z} z\right)+\cos \left(\bar{h}_{x} x-\bar{h}_{z} z\right)\right)
$$

where $\bar{h}_{x}=\bar{h}_{z}=2 \pi / d_{x, z}$ are the projections of translational vectors $\vec{K}^{ \pm}=\bar{h}_{x} \vec{x}_{0} \pm \bar{h}_{z} \vec{z}_{0}$ of the two-dimensional grating in the $x$ and $z$ directions and $d_{x, z}$ are the periods of corresponding modulations. The area with modulation has the length $l_{z}$ on the longitudinal $z$ axis and width $l_{x}$ on the transverse (lateral) coordinate $x$. This area forms a 2D Bragg resonator.

The fields of TM-polarized eigenmodes of a 2D Bragg resonator [6,7] are defined as the sum of four partial wave fluxes

$$
E_{y}=\operatorname{Re}\left(f(y)\left(\begin{array}{l}
C_{z}^{+}(x, z) e^{-i \bar{h} z}+C_{z}^{-}(x, z) e^{i \bar{h} z}+ \\
C_{x}^{+}(x, z) e^{-i \bar{h} x}+C_{x}^{-}(x, z) e^{i \bar{h} x}
\end{array}\right) e^{i \omega t}\right)
$$


propagating in the $\pm x$ and $\pm z$ directions. Here $C_{x, z}^{ \pm}$are the complex amplitudes slowly varying on the wavelength scale, $f(y)$ is the function determining the wave structure in the direction normal to the waveguide surface, which coincides with one of the TM-modes of the indicated waveguide, $E_{y}$ is the $y$ component of the electric field. In this case, the average waveguide thickness is selected so that a single mode propagates in the operatingfrequency range.

At the Bragg structure given by relation (2), each wave beam under Bragg resonance conditions is scattered in two mutually opposite directions, perpendicularly to the initial propagation direction. Mutual scattering of wave beams under these conditions is described by the equations [6]

$$
\begin{aligned}
& \pm \frac{\partial C_{z}^{ \pm}}{\partial z}+i \delta C_{z}^{ \pm}+i \alpha \bar{h}\left(C_{x}^{+}+C_{x}^{-}\right)=0, \\
& \pm \frac{\partial C_{x}^{ \pm}}{\partial x}+i \delta C_{x}^{ \pm}+i \alpha \bar{h}\left(C_{z}^{+}+C_{z}^{-}\right)=0,
\end{aligned}
$$

where $\delta=h(\omega)-\bar{h}$ is the Bragg resonance deturning

$$
\alpha=\frac{c \bar{h} b_{1}}{4 \omega} \frac{\left(\varepsilon-\bar{h}^{2} c^{2} / \omega^{2}\right)\left(1+\varepsilon^{-2}\right)}{\left(\bar{h}^{2} c^{2} /\left(\varepsilon \omega^{2}\right)+\bar{h}^{2} c^{2} / \omega^{2}+1\right) b_{0}+2\left(\bar{h}^{2}-\omega^{2} / c^{2}\right)^{-1 / 2}}
$$

is the coupling coefficient of partial waves. We note that the wavenumber and frequency $\omega$ are bound by the dispersion relation of the lowest mode of the dielectric waveguide. It should also be noted that wavebeam diffraction was neglected in deriving Eq. (3) under the assumption that the corresponding Fresnel parameters are rather high.

The spectrum of eigenmodes of the 2D Bragg resonator can be determined by solving the boundary-value problem for which Eqs. (3) should be complemented by the boundary conditions

$$
\left.C_{z}^{+}\right|_{z=0}=0,\left.C_{z}^{-}\right|_{z=l_{z}}=0,\left.C_{x}^{+}\right|_{x=0}=0,\left.C_{x}^{-}\right|_{x=l_{x}}=0
$$

which correspond to the absence of external energy fluxes.

Under conditions of strong waves coupling, $\alpha l_{x, z}>>1$, solutions for complex $\delta_{n, m}$ which define the spectrum of eigenfrequencies

$$
\omega_{n, m} \approx c \bar{h}+c \operatorname{Re} \delta_{n, m}
$$

and mode Q-factors of the 2D Bragg resonator,

$$
Q_{n, m} \approx \bar{h} /\left(2 \operatorname{Im} \delta_{n, m}\right)
$$

are given by the relations [6],

$$
\begin{gathered}
\delta_{n, m}= \pm \frac{\pi^{2} m n}{2 \alpha l_{z} l_{x}}+i \frac{\pi^{2}}{2 \alpha^{2} l_{z} l_{x}}\left(\frac{n^{2}}{l_{z}}+\frac{m^{2}}{l_{x}}\right), \\
\delta_{n, m}= \pm\left[2 \alpha+\frac{\pi^{2}}{4 \alpha}\left(\frac{n^{2}}{l_{z}^{2}}+\frac{m^{2}}{l_{x}^{2}}\right)\right]+i \frac{\pi^{2}}{2 \alpha^{2}}\left(\frac{n^{2}}{l_{z}^{3}}+\frac{m^{2}}{l_{x}^{3}}\right),
\end{gathered}
$$

where $n$ and $m$ are the mode indices for longitudinal $z$ and transverse $x$ coordinates, respectively. According to Eqs. (4), (5) there are two sets of high-Q modes (Fig. 2). One of them is near the Bragg frequency; the second is near the band-gap edge. 


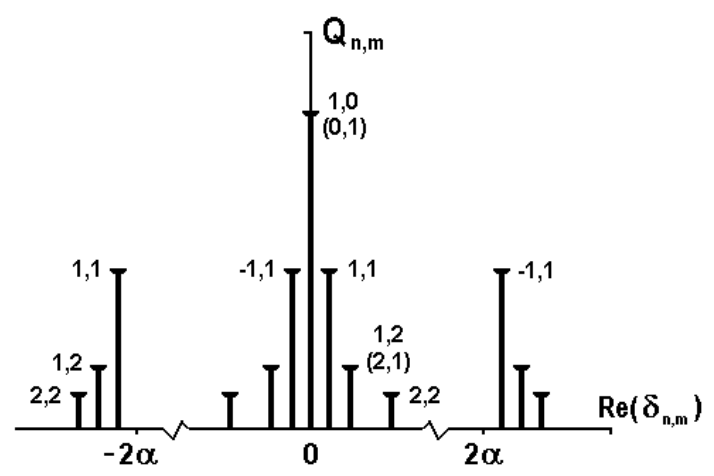

Fig. 2. Spectrum of modes of the two-dimensional Bragg resonator, determined within the coupled wave method (eqs. (4), (5)).

\section{Simulation of the selective characteristics of dielectric two-dimensional Bragg resonators}

A theoretical analysis of two-dimensional Bragg resonators was carried out using averaged models developed at the IAP RAS based on the coupled wave method. To verify the applicability of these models, a 3D simulations of these systems was performed based on the commercial code CST Microwave Studio.

(a)

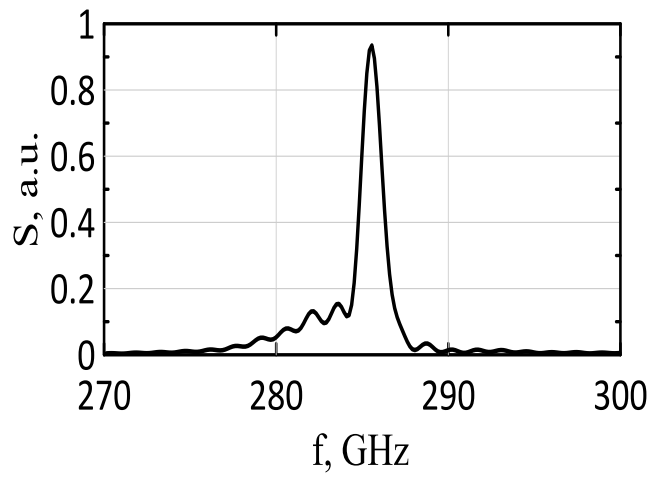

(b)

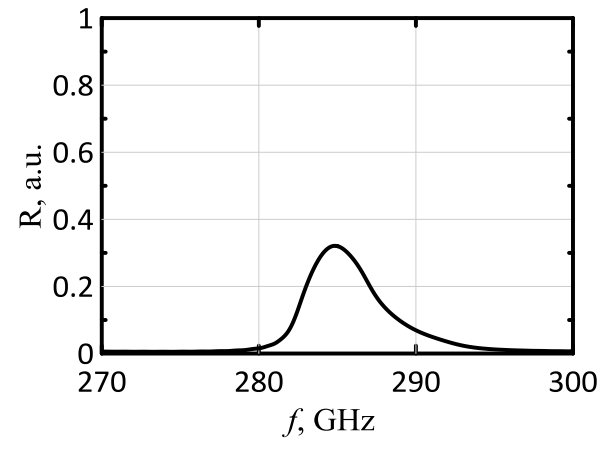

Fig. 3. Results of the CST simulation of two-dimensional Bragg structure excitation by a short electromagnetic pulse. (a) The field spectra in the final stage of the perturbation decay process corresponds to selective excitation of fundamental mode. (b) Frequency dependence of integral reflection coefficient $\mathrm{R}$ in the case of normal incidence of plane wave.

Based on the theoretical analysis, 2D G-band Bragg structures based on Visijet plastics with dielectric constant $\operatorname{Re} \varepsilon=1.8$ and loss tangent $\tan \delta=0.035$ have been developed and manufactured by $3 \mathrm{D}$ printers. The complex permittivity was measured in the longwavelength part of the millimeter range $(30-40 \mathrm{GHz})$, which simplifies the manufacture of experimental models. The data obtained were used in numerical modeling in the higher frequency $\mathrm{G}$ range. Figure 3 presents the results of modeling a 2D structure with periods of $d_{x}=d_{z}=1 \mathrm{~mm}$, thickness of $b_{0}=0.2 \mathrm{~mm}$, and longitudinal and transverse dimensions $l_{z, x}=10 \mathrm{~cm}$. According to the simulations, this structure had a working Bragg scattering zone in the vicinity of $285 \mathrm{GHz}$. 
Thus, the location of the fundamental mode and the absolute value of its Q-factor, found by direct numerical simulation, are in good agreement with analytical solutions. It is more important that the existence of this mode at the center of the Bragg resonance zone has been demonstrated.

\section{Elaboration of the experimental setup for investigation of dielectric 2D Bragg structures for $\mathrm{THz}$ band}

For experimental investigations of an oversized planar dielectric 2D Bragg structure, an excitation of this structure by a wide wavebeam with flat phase front should be provided. 2D planar waveguided comprises parallel metallic plates with radiation propagating in between.

(a)
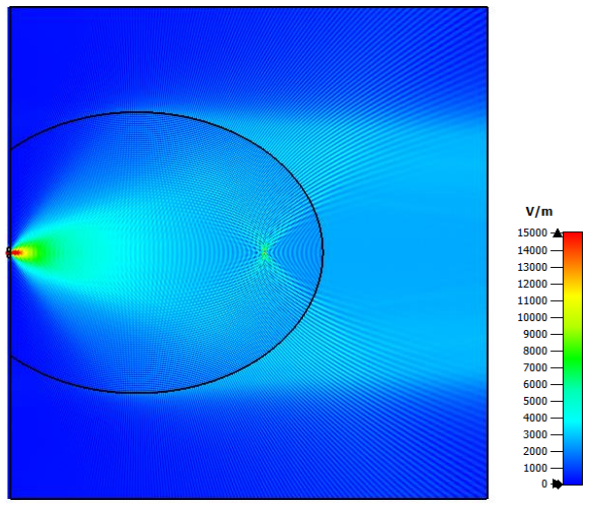

(b)
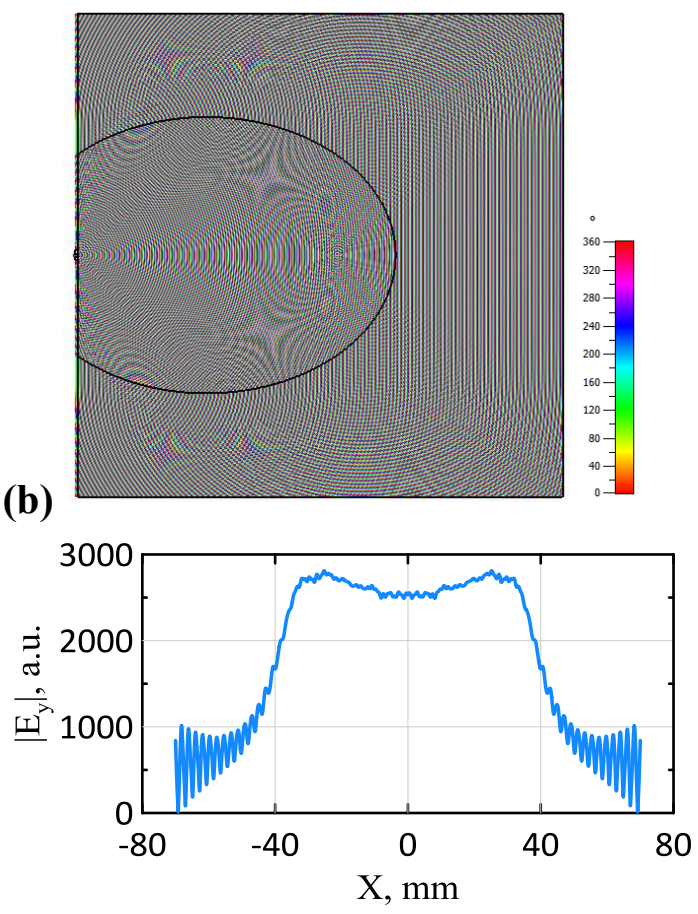

(c)

Fig. 4. Results of simulation of the exciter based on the ellipse-shaped dielectric insert. Feeding single-mode waveguide is located in a focus of the ellipse. (a) Amplitude and (b) phase Ey field distribution, (c) output amplitude distribution at $285 \mathrm{GHz}$. 
In case of simple excitation of such structure by a single-mode rectangular waveguide, the phase front is curved and the amplitude strongly depends on the transverse (with respect to propagation direction) coordinate. In order to obtain a flat phase front and a distribution as close to constant as possible, a dielectric insert shown in Fig.4a could be used. The insert has an elliptic shape with one of the ellipse's focuses located in the middle of the feeding single-mode waveguide. In case, when the ratio between the ellipse axis is equal to $\sqrt{1-\varepsilon^{-1}}$ ( $\varepsilon$ is the dielectric permittivity), the radiation outcoming from the dielectric would have amplitude close to the constant in the center (Fig.4b). This property does not depend on the radiation frequency if the dimensions of the insert are much larger than the wavelength. In the vicinity of the insert, the field structure is distorted because the part of the radiation is reflected from the inside boundary of the waveguide and comes out from the opposite side. However, this radiation is mainly directed to the side, so at some distance from the boundary the field structure is substantially improved (Fig.4c). For the width of the insert of $80 \mathrm{~mm}$ and aperture of $50 \mathrm{~mm}$, the field non-uniformity does not exceed $10 \%$.

(a)

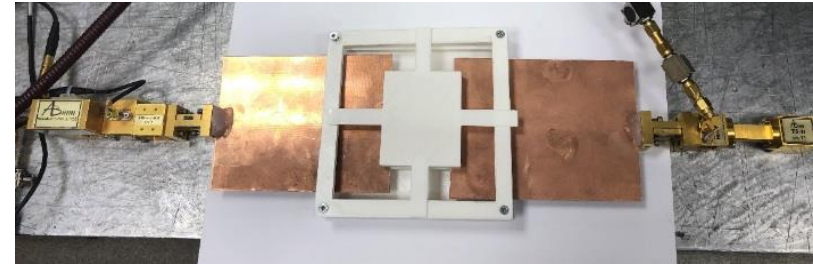

(b)

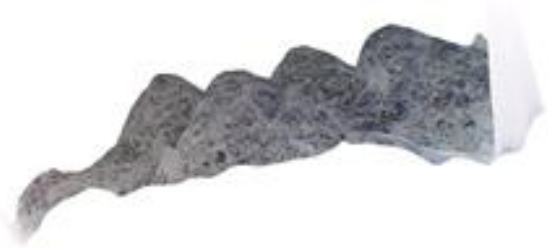

Fig. 5. (a) Experimental setup for measuring the reflection, transmission and transverse scattering coefficients of the dielectric structure with 2D corrugation. (b) A photo of the surface of the manufactured 2D structure.

Selective properties of the prototypes of the described structures were verified experimentally in "cold" electrodynamic tests with Fresnel parameter values of $F_{x, z} \sim 100$. Experiments have demonstrated good coincidence with simulations in terms of location and width of the Bragg waveband and in terms of amplitude characteristics of integral scattering coefficients.

\section{Conclusions}

Thus, the conducted theoretical and experimental investigations have confirmed the viability of $2 \mathrm{D}$ dielectric Bragg structures for $\mathrm{THz}$ frequency band. A widely available and relatively cheap technique of manufacturing such structures was elaborated using 3D printing method. Good coincidence of electrodynamical characteristics of 2D periodic structures measured in "cold" tests with those obtained in simulations with commercial software package. The conducted experimental investigations have confirmed that the dielectric properties of plastics used in the proposed technique are adequate for manufacturing the dielectric $2 \mathrm{D}$ structure prototypes with manufacturing accuracy acceptable for $\mathrm{THz}$ frequency band. 
This work was supported in the framework of IAP RAS government task No. 0035-2019-0001 RFBR project No. 18-48-520022.

\section{References}

1. A. V. Arzhannikov, N. S. Ginzburg, V.S. Nikolaev, N. Yu. Peskov, A. S. Sergeev, S.L.Sinitsky, R. P. Zotkin, and M. V. Yushkov, in Abstracts of the 14th International FEL Conference, Kobe, Japan (Kobe University, 1992)

2. N. S. Ginzburg, N. Yu. Peskov, and A. S. Sergeev, Opt. Commun. 112, 151 (1994).

3. A. V. Arzhannikov, N. S. Ginzburg, V.G. Ivanenko, I. A. Ivanov, P. V. Kalinin, A. S. Kuznetsov, S. A. Kuznetsov, N.Yu. Peskov, A. S. Sergeev, S. L. Sinitsky, V.D. Stepanov, and V. Yu. Zaslavsky, JEPT Lett. 87, 618 (2008)

4. I. V. Konoplev, A. W. Cross, A. D. R. Phelps, W. He, K. Ronald, C. G. Whyte, C.W. Robertson, N. S. Ginzburg, N. Yu. Peskov, A. S. Sergeev, and M. Thumm, Phys. Rev. E 76, 056406 (2007)

5. D. A. Yanson, E. U. Rafailov, G. S. Sokolovskii, V. I. Kuchinskii, A. C. Bryce, J. Marsh, and W. Sibbett, J. Appl. Phys. 95, 1502 (2004)

6. N.S. Ginzburg, V.R. Baryshev, A.S. Sergeev, A.M. Malkin, Phys. Rev. A. 91, 053806 (2015)

7. V.R. Baryshev, N.S. Ginzburg, V.Yu. Zaslavsky, A.M. Malkin A.S. Sergeev, Quantum Electronics, 39(5), 463 (2009) 